\title{
Structure and Characterization of an Americium
}

\section{$\operatorname{Bis}\left(O, O^{\prime}\right.$-diethyl $)$ dithiophosphate Complex}

R. D. M. Greer, Cristian Celis-Barros, Joseph M. Sperling, Alyssa N. Gaiser, Cory J. Windorff, Thomas E. Albrecht-Schönzart*

Department of Chemistry and Biochemistry, Florida State University, Tallahassee, Florida 32306

*talbrechtschoenzart@gmail.com

\section{$\underline{\text { Supporting Information }}$}

\section{Table of Contents}

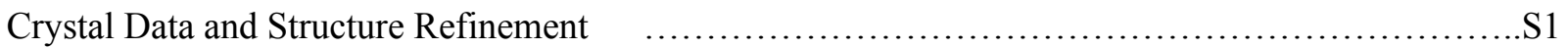

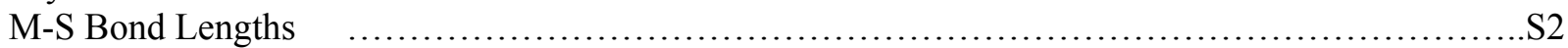

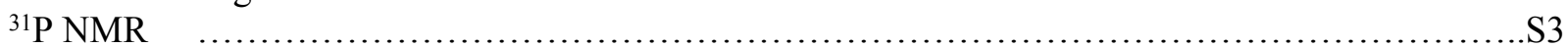

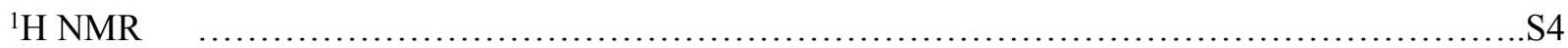

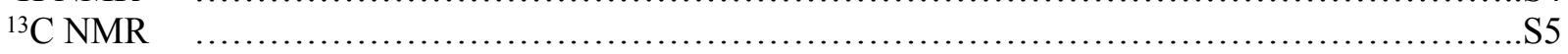

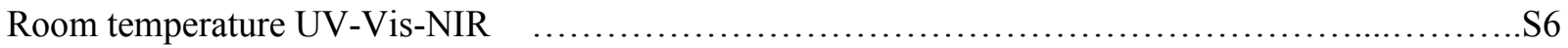

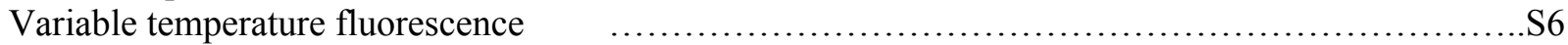

\section{Crystal data and Structure Refinement for AmDTPEt}

\begin{tabular}{|l|l|}
\hline Chemical formula & {$\left[\mathrm{As}\left(\mathrm{C}_{6} \mathrm{H}_{5}\right)_{4}\right] \mathrm{Am}\left[\mathrm{S}_{2} \mathrm{P}\left(\mathrm{OC}_{2} \mathrm{H}_{5}\right)_{2}\right]_{4}$} \\
\hline Empirical formula & $\mathrm{C}_{40} \mathrm{H}_{60} \mathrm{P}_{4} \mathrm{~S}_{8} \mathrm{O}_{8} \mathrm{AsAm}$ \\
\hline Formula weight & 1367.16 \\
\hline Temperature & $100 \mathrm{~K}$ \\
\hline Crystal size $(\mathrm{mm})$ & $0.682 \times 0.381 \times 0.35$ \\
\hline Crystal system & Monoclinic \\
\hline Space group & $P 2_{1 / \mathrm{c}}$ \\
\hline$a(\AA)$ & $19.4974(9)$ \\
\hline$b(\AA)$ & $15.0233(7)$ \\
\hline$c(\AA)$ & $19.7713(9)$ \\
\hline$\beta$ & $110.8640(10)$ \\
\hline$V\left(\AA^{3}\right)$ & $5412.2(4)$ \\
\hline$Z$ & 4 \\
\hline$D_{\text {calc. }}$ & 1.678 \\
\hline$\mu($ Mo K $\alpha)($ nm $)$ & 0.71073 \\
\hline$F(000)$ & 2720 \\
\hline$\theta$ range & $2.204-30.797$ \\
\hline Completeness & $99.9 \%$ \\
\hline Reflections collected & 16848 \\
\hline Unique reflections & 13854 \\
\hline Parameters refined & 567 \\
\hline$R_{l}, w R_{2}[I>2 \sigma(I)]$ & $0.0315,0.0653$ \\
\hline$R_{l}, w R_{2}$ all reflns. & $0.0470,0.0722$ \\
\hline GOOF on $F^{2}$ & 1.066 \\
\hline
\end{tabular}

\section{Table S1}




\section{Crystal data and Structure Refinement for NdDTPEt}

\begin{tabular}{|l|l|}
\hline Chemical formula & {$\left[\mathrm{As}\left(\mathrm{C}_{6} \mathrm{H}_{5}\right)_{4}\right] \mathrm{Nd}\left[\mathrm{S}_{2} \mathrm{P}\left(\mathrm{OC}_{2} \mathrm{H}_{5}\right)_{2}\right]_{4}$} \\
\hline Empirical formula & $\mathrm{C}_{40} \mathrm{H}_{60} \mathrm{P}_{4} \mathrm{~S}_{8} \mathrm{O}_{8} \mathrm{AsNd}$ \\
\hline Formula weight & 1268.40 \\
\hline Temperature & $100 \mathrm{~K}$ \\
\hline Crystal system & Monoclinic \\
\hline Space group & $P 2_{1 / \mathrm{c}}$ \\
\hline$a(\AA)$ & $19.5348(11)$ \\
\hline$b(\AA)$ & $15.0318(9)$ \\
\hline$c(\AA)$ & $19.7917(12)$ \\
\hline$\beta$ & $110.800(2)$ \\
\hline$V\left(\AA^{3}\right)$ & $5432.9(6)$ \\
\hline$Z$ & 4 \\
\hline$\mu($ Mo Ka) (nm) & 0.71073 \\
\hline$F(000)$ & 2580 \\
\hline$\theta$ range & $2.202-29.644$ \\
\hline Completeness & $99.9 \%$ \\
\hline Reflections collected & 15380 \\
\hline Unique reflections & 13556 \\
\hline Parameters refined & 567 \\
\hline$R_{l}, w R_{2}[I>2 \sigma(I)]$ & $0.0337,0.0678$ \\
\hline$R_{l}, w R_{2}$ all reflns. & $0.0421,0.0720$ \\
\hline GOOF on $F^{2}$ & 1.157 \\
\hline
\end{tabular}

Table S2

M-S Bond Lengths for AmDTPEt and NdDTPEt

\begin{tabular}{|l|c|c|c|}
\hline & Am-S Bond Length & Nd-S Bond Length & AM-S (Nd-Am) \\
\hline S1 & $2.8866(7)$ & $2.9047(7)$ & $0.0181(7)$ \\
\hline S2 & $2.8915(8)$ & $2.9066(7)$ & $0.0151(8)$ \\
\hline S3 & $2.8943(8)$ & $2.9162(7)$ & $0.0219(8)$ \\
\hline S4 & $2.8976(9)$ & $2.9169(8)$ & $0.0193(9)$ \\
\hline S5 & $2.8994(8)$ & $2.9214(7)$ & $0.0220(8)$ \\
\hline S6 & $2.9345(8)$ & $2.9432(7)$ & $0.0087(8)$ \\
\hline S7 & $2.9441(7)$ & $2.9546(7)$ & $0.0105(7)$ \\
\hline S8 & $2.9448(8)$ & $2.9569(7)$ & $0.0121(8)$ \\
\hline Average & $2.9116(8)$ & $2.9275(8)$ & $0.0159(8)$ \\
\hline
\end{tabular}

Table S3 
${ }^{31}$ P NMR for AmDTPEt

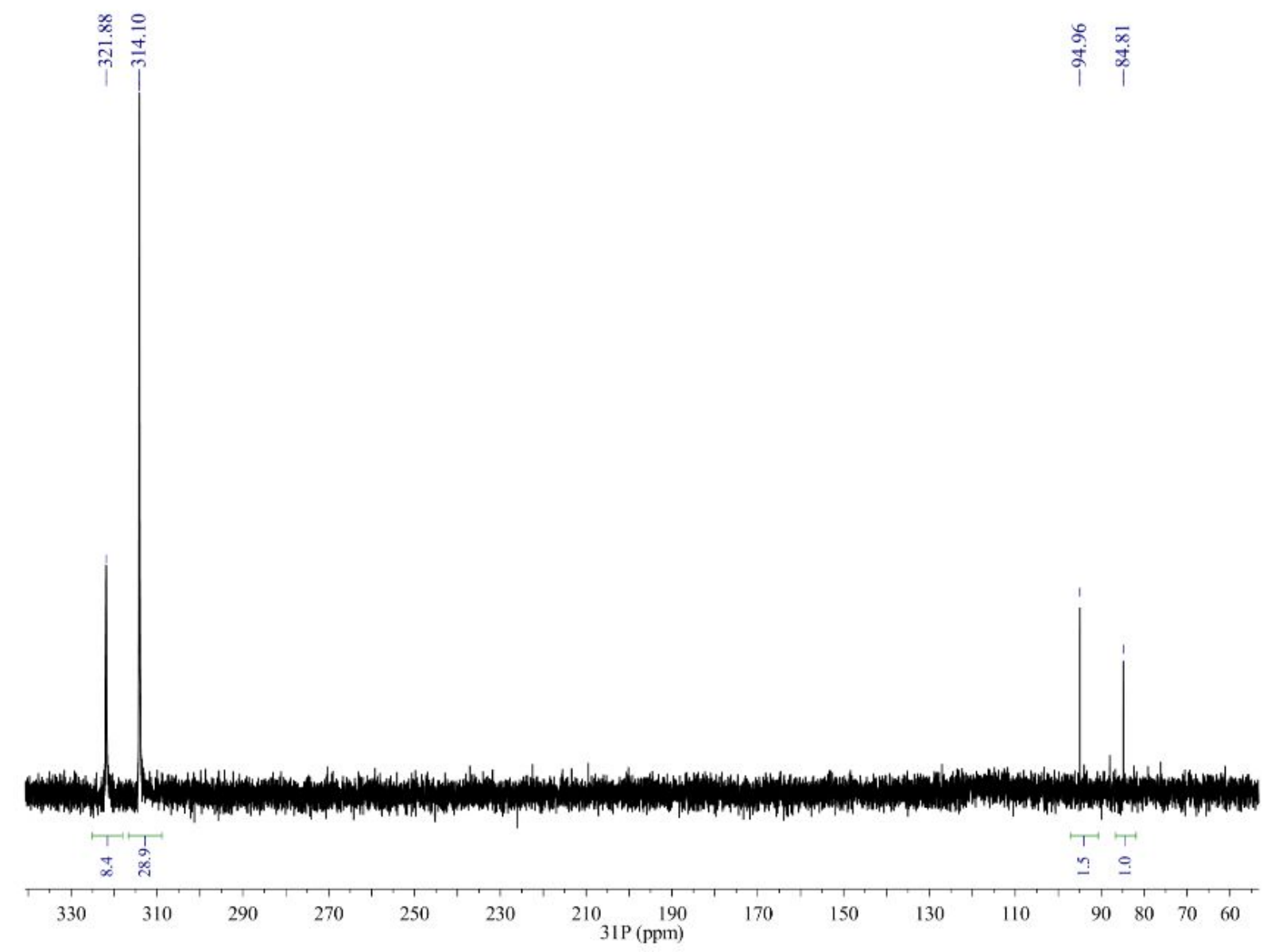

Figure $\mathrm{S1}$, $161.99 \mathrm{MHz}^{31} \mathrm{H} \mathrm{NMR} \mathrm{of} \mathrm{AmDTPEt} \mathrm{in} \mathrm{CDCl}_{3}$, external 85\% $\mathrm{H}_{3} \mathrm{PO}_{4}$ reference 


\section{${ }^{1} \mathrm{H}$ NMR for AmDTPEt}

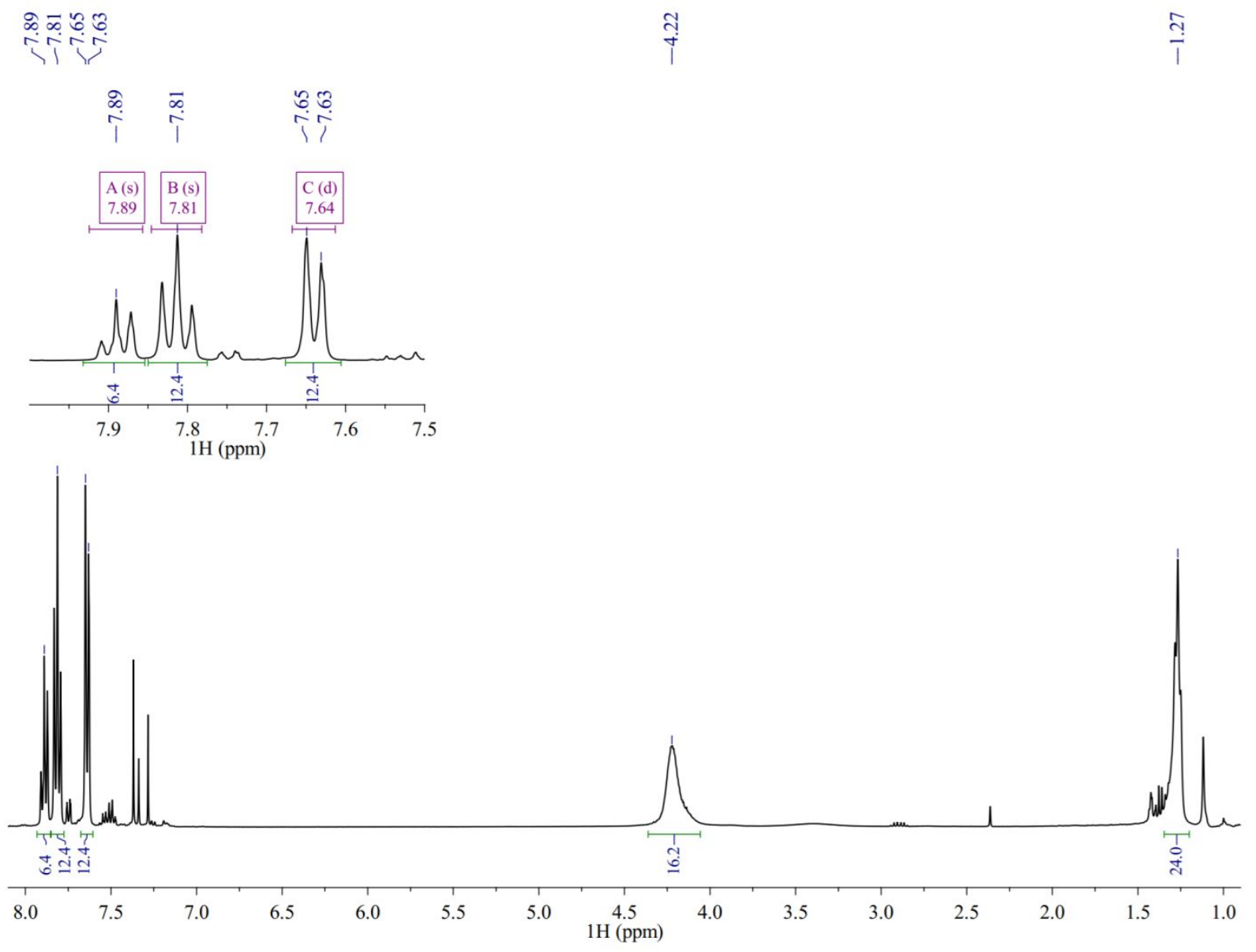

Figure S2, $400 \mathrm{MHz}^{1}{ }^{1} \mathrm{H} \mathrm{NMR}$ of AmDTPEt in $\mathrm{CDCl}_{3}$, internal solvent reference 
${ }^{13}$ C NMR for AmDTPEt
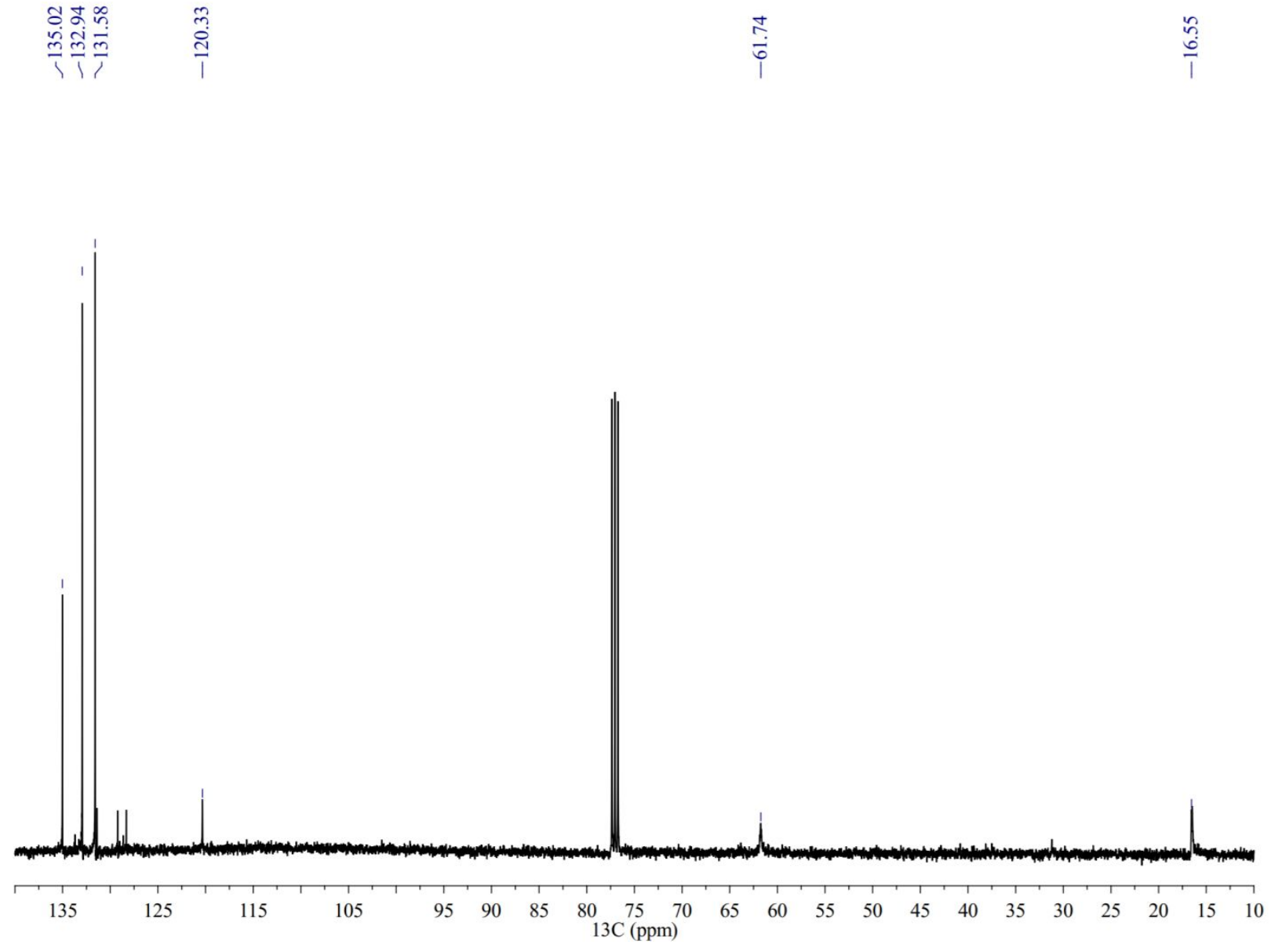

Figure S3, $400 \mathrm{MHz}^{13} \mathrm{C} N \mathrm{NR}$ of AmDTPEt in $\mathrm{CDCl}_{3}$, internal solvent reference 
Room temperature absorbance for AmDTPEt

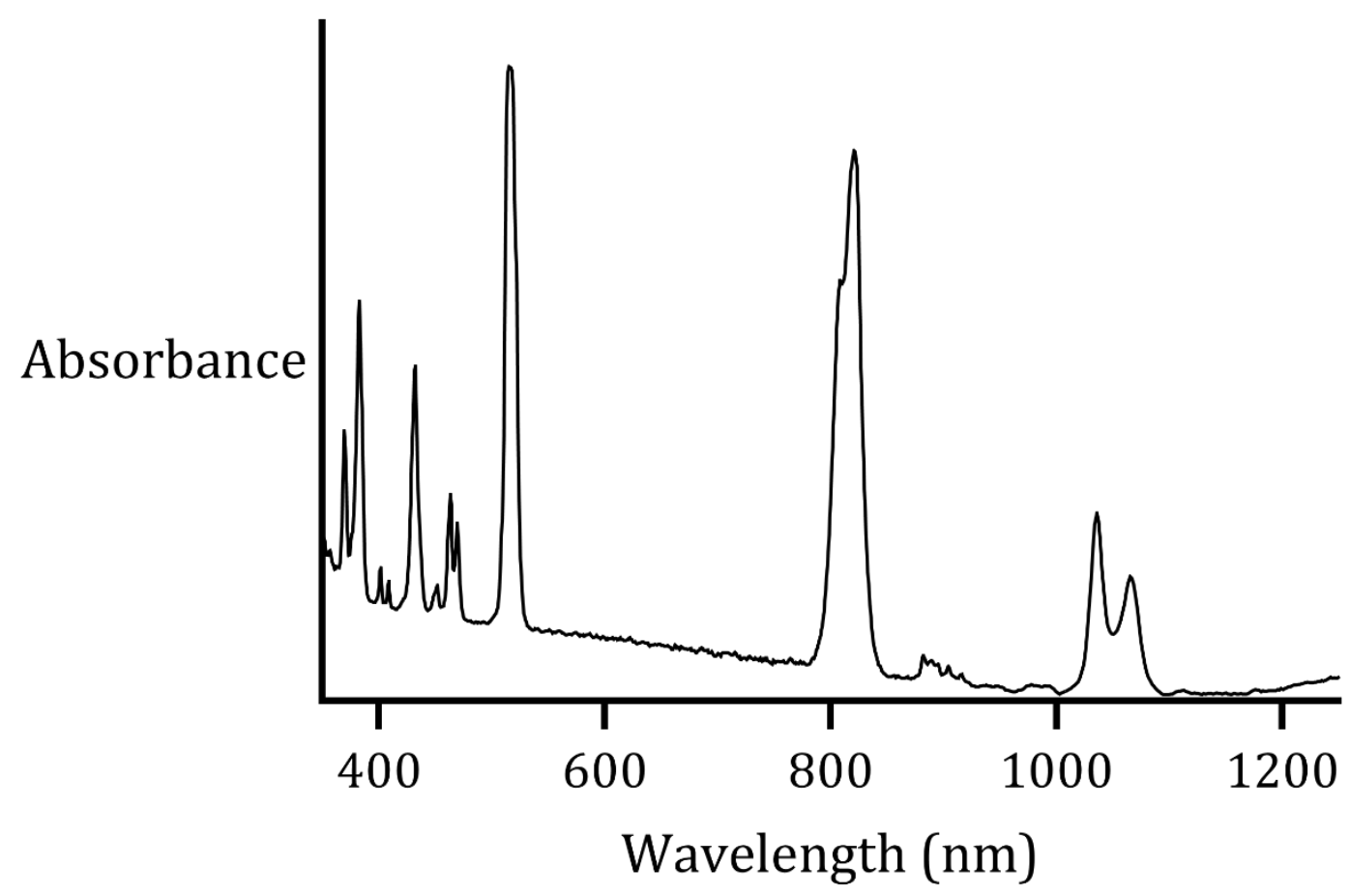

Figure S4 UV-visible-NIR absorbance collected on an AmDTPEt crystal at room temperature.

Variable Temperature Fluorescence for AmDTPEt

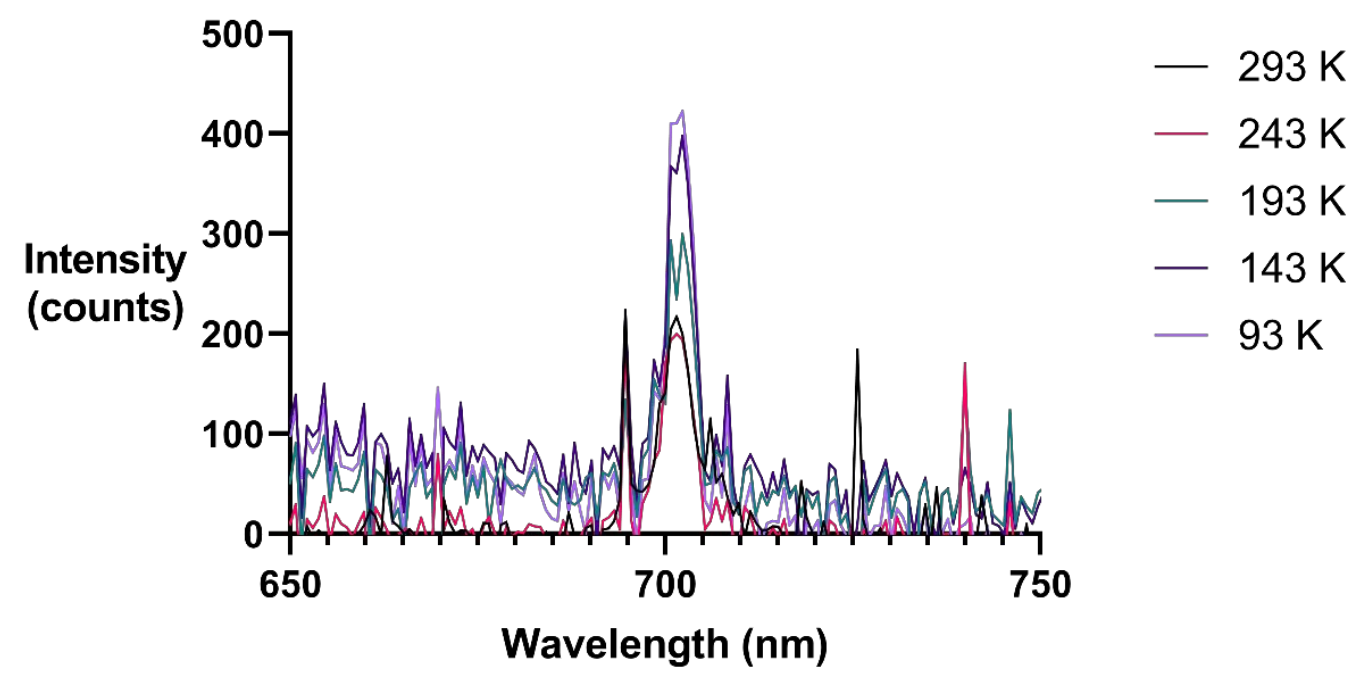

Figure S5 Variable temperature fluorescence spectra of AmDTPEt crystals, with excitation at $365 \mathrm{~nm}$. 